\title{
Tubulointerstitial nephritis and uveitis syndrome post-COVID-19: a paediatric case and literature review
}

\author{
Maria Cristina Maggio ${ }^{1}$, Filippo Collura ${ }^{1}$, Maria Michela D'Alessandro², Barbara \\ Gramaglia $^{1}$, and Giovanni Corsello ${ }^{1}$ \\ ${ }^{1}$ University of Palermo \\ ${ }^{2}$ ARNAS Palermo
}

January 13, 2022

\section{Tubulointerstitial nephritis and uveitis syndrome post-COVID-19: a paediatric case and liter- ature review}

To the Editor.

The angiotensin-converting-enzyme-2 receptor is expressed in many extrapulmonary organs: eyes (conjunctival, corneal and limbal epithelial cell, retina), nerves, vessels, enterocytes of the small intestine, the kidney proximal tubules, with possible involvement of these districts by SARS-CoV-2 infection. Positive conjunctival swab tests are documented, as well (1). Furthermore, a transmembrane serine protease (TMPRSS2) cleaves the S protein into S1 and S2 domains upon virus binding to ACE2 and consequently allows the fusion of the viral membrane with the host cellular membranes permitting the viral entry. The cornea is a potential site for SARS-CoV-2 infection: the co-expression of ACE2 and TMPRSS2 are documented in corneal epithelium and endothelium. However, the expression of both ACE2 and TMPRSS2 in superficial conjunctival epithelium is much lower than in lung and kidney tissues, as only $6.6 \%$ of cells of the superficial conjunctival epithelium express both entry proteins TMPRSS2 and ACE2 . Recently, some cases of uveitis and optic neuritis as an uncommon presentation of ocular involvement in COVID-19 were described (2). Furthermore, the phlogistic involvement of renal interstitium and uveal tissue characterizes the two components of tubulointerstitial nephritis and uveitis (TINU) syndrome. Eyes and kidneys involvement can persist as asymptomatic and can have an independent outcome. TINU usually shows bilateral anterior uveitis and may evolve into a chronic disease. TINU syndrome accounts for approximately $1 \%-2 \%$ of uveitis in tertiary referral centres (3). Renal and ocular manifestations in some patients do not occur simultaneously, making the diagnosis challenging. Treatment includes topical and oral corticosteroids. Renal disease shows as acute kidney injury and resolves spontaneously with full recovery of kidney function or evolves into permanent renal insufficiency. However, uveitis can persist, or it recurs years after its first presentation (4).

A 7-years-old girl was admitted in our Children Hospital for persistent fever and bilateral red eyes, photophobia, eye pain, during the COVID-19 pandemic. The nasopharyngeal swab for SARS-CoV-2, diagnosed by a reverse transcription-polymerase chain reaction - RT-PCR diagnostic on 2 molecular targets (E, N2) (Xpert Xpress SARS-CoV-2, Cepheid, Maurens-Scopont, France), was positive in two different swabs, confirming SARS-CoV-2 infection. The swab for other respiratory viruses was negative. The ophthalmologist diagnosed bilateral, anterior uveitis and she started a course of eye drops $0.1 \%$ dexamethasone (to both eyes at an initial schedule of once every $2 \mathrm{~h}$ for 3 days), and a 1-week course of eye drops to both eyes twice daily. Treatment was tapered over a 2-week period and the patient's symptoms resolved. Furthermore, she showed nocturia, polyuria and polydipsia.

14 days after the end of steroids' treatment and a negative swab, she showed a relapse of uveitis, with a 
reduction of visual acuity in the right eye (4/10), ipsilateral conjunctival hyperaemia, bilateral irido-capsular synechiae, papilledema and macular edema. The optical computerized tomography (OCT) confirmed the diagnosis. The encephalic computerized axial tomography was negative, and the magnetic resonance showed a bilateral slight distension of the perioptic sheath in the retrobulbar area. Cerebrospinal fluid (CSF) analysis showed normal chemical-physical examination and cell count, with concordance between the CSF and serum bands, excluding an intrathecal production of IgG. Specific PCR analysis for viruses and microbic agents was conducted on CSF and was negative. Furthermore, autoimmune tests, the anti-NMDA, anti-MOG and anti-aquaporin 4 antibodies were negative. Blood tests revealed: C-reactive protein (CRP) $1.79 \mathrm{mg} / \mathrm{dl}$ (n.v. < 0.5), erythrocyte sedimentation rate $($ ESR) $56 \mathrm{~mm} / \mathrm{h}$, serum amyloid A (SAA) $30 \mathrm{ng} / \mathrm{dl}(<6.4)$, fibrinogen $460.3 \mathrm{mg} / \mathrm{dl}$, ferritin, IL-6, lymphocyte subpopulation analysis in the normal range, creatinine $0.83 \mathrm{mg} / \mathrm{dl}$; hyponatremia: $134 \mathrm{mmol} / \mathrm{L}$; mild increase of C3: $150 \mathrm{mg} / \mathrm{dl}$ (n.v. < 140); blood urea and $\mathrm{C} 4$ were in the normal range. The kidney involvement was confirmed by the creatinine values above the normal range $(0.83 \mathrm{mg} / \mathrm{dl})$, with a glomerular filtration rate of $60 \mathrm{ml} / \mathrm{min} / 1.73 \mathrm{~m} 2$, according to Scwartz's formula. The chemical-physical examination of the urine shows glycosuria $(172 \mathrm{mg} / \mathrm{dl})$, albuminuria (101 $\mathrm{mg} / \mathrm{l})$, proteinuria $(130 \mathrm{mg} / \mathrm{dl})$. The diagnosis of tubulointerstitial nephritis was done and confirmed by the increased serum and urinary levels of tubular enzymes beta2 microglobulin, alpha1 microglobulin, alpha2 macroglobulin. The Human Leukocyte Antigens (HLA) -B27, -B51, -B57 were not expressed in the patient. Specific anti-SARS-CoV-2 IgG were significantly increased: $8080.7 \mathrm{UA} / \mathrm{ml}$ (n.v. < 50). She was treated with prednisone $(1 \mathrm{mg} / \mathrm{kg} /$ day $)$ for $1 \mathrm{month}$, with a gradual tapering of the steroid dose.

She started a second course of topic $0.1 \%$ dexamethasone and mydriatics.

During the follow-up, creatinine and glomerular filtration rate normalized, with the resolution of uveitis, as well.

However, 3 months after the stop of steroids, she showed a relapse of bilateral uveitis, treated again with steroids (prednisone: $0.75 \mathrm{mg} / \mathrm{kg} /$ day) for 1 month, with a gradual tapering. A new relapse occurred 2 months later, and the patient started anti-TNF-alfa treatment (adalimumab) (40 mg/every 14 days), with the complete resolution of the eye involvement. The diagnosis of TINU syndrome was done, in conformity of the clinical outcome of the patient.

TINU syndrome is a rare disease with kidney and eyes inflammatory involvement. The prevalence of TINU syndrome between patients attending specialist uveitis centres, is $<0.1-2 \%$ in the general populations and 1.1-2.3\% in paediatric populations (5), with variation reflecting many factors including level of diagnostic certainty necessary to recognize the disease, otherwise underdiagnosed. The epidemiological and etiological data do not permit the real definition of genetic, epigenetic and environmental factors implicated in the pathogenesis of the disease (5).

The limited number of patients and studies do not support the identification of a HLA haplotype and a genetic pattern that predispose to TINU syndrome. Some studies propose infectious triggers or drugs as the principal acquired risk factors for the syndrome. Furthermore, there is an evident different approach between studies on renal diseases, highlighting the role of a drug-induced hypersensitivity reaction in most of the cases, and ophthalmic studies, with no clear supported triggers (5). The most frequent drugs correlated to TINU syndrome are antibiotics and non-steroidal anti-inflammatory drugs. It is essential to exclude systemic diseases proven to trigger an overlap of ocular and renal inflammation, especially Behçet disease, systemic lupus erythematosus (SLE), Sjogren's syndrome, sarcoidosis and tuberculosis (TB), atypical Kawasaki disease (6-9). COVID-19 and MIS-C can show eyes involvement. Ocular symptoms in adults with COVID-19 are polymorphous: conjunctival hyperaemia, epiphora, chemosis, anterior uveitis, visual impairment, abducens nerve palsy. Most of children with MIS-C or with Kawasaki disease, show conjunctivitis, less frequently anterior uveitis or corneal punctate epitheliopathy (10). COVID-19-related uveitis show multifactorial pathogenetic mechanisms, including: 1) direct cytopathic effect of the virus: the ACE-2 and TMPRSS2 are in the cornea and limbus; 2) the eyes thrombotic vasculopathy; 3) the antigen mimicry between virus and self-antigens of the eyes, especially in patients with HLA-B27; 4) activation of the cytokine secretion, especially of TNF-alfa, IL-1, IL-18. 
The clinical course of our patient showed a recurrence of uveitis, with full recovery of kidney function after a course of steroids therapy. However, uveitis recurred several times, after its initial presentation, and resolved with adalimumab.

The prompt diagnosis and treatment of uveitis is of great consequence, due to the unfavourable ocular complications, as band keratopathy, posterior synechiae, cataract, increased intraocular pressure with secondary glaucoma, cystoid macular oedema, visual impairment. To our knowledge, this is the first paediatric case with TINU syndrome and SARS-CoV-2 in the international literature.

Keywords: tubulointerstitial nephritis and uveitis (TINU) Syndrome; SARS-CoV-2; adalimumab; COVID-19.

References

1) Zhong Y, Wang K, Zhu Y, Lyu D, Yu Y, Li S, Yao K. Ocular manifestations in COVID-19 patients: A systematic review and meta-analysis. Travel Med Infect Dis. 2021 Nov-Dec;44:102191. doi: 10.1016/j.tmaid.2021.102191. Epub 2021 Nov 8. PMID: 34763068; PMCID: PMC8574127. 2) Boz AAE, Atum M, Çakır B, Karabay O, Çelik E, Alagöz G. Outcomes of the Ophthalmic Examinations in Patients Infected by SARS-CoV-2. Ocul Immunol Inflamm. 2021 May 19;29(4):638-641. doi: 10.1080/09273948.2020.1844904. Epub 2020 Nov 23. PMID: 33226284. 3) Amaro D, Carreño E, Steeples LR, Oliveira-Ramos F, MarquesNeves C, Leal I. Tubulointerstitial nephritis and uveitis (TINU) syndrome: a review. Br J Ophthalmol. 2020 Jun;104(6):742-747. doi: 10.1136/bjophthalmol-2019-314926. Epub 2019 Nov 12. PMID: 31719109. 4) Pereira C, Costa-Reis P, Esteves da Silva J, Stone R. A child with tubulointerstitial nephritis and uveitis (TINU) syndrome. BMJ Case Rep. 2018 Feb 17;2018:bcr2017222766. doi: 10.1136/bcr-2017-222766. PMID: 29455181; PMCID: PMC5836612. 5) Okafor LO, Hewins P, Murray PI, Denniston AK. Tubulointerstitial nephritis and uveitis (TINU) syndrome: a systematic review of its epidemiology, demographics and risk factors. Orphanet J Rare Dis. 2017 Jul 14;12(1):128. doi: 10.1186/s13023-017-0677-2. PMID: 28709457; PMCID: PMC5513333.) 6) Gallizzi R, Pidone C, Cantarini L, Finetti M, Cattalini M, Filocamo G, Insalaco A, Rigante D, Consolini R, Maggio MC, Civino A, Martino S, Olivieri AN, Fabio G, Pastore S, Mauro A, Sutera D, Trimarchi G, Ruperto N, Gattorno M, Cimaz R. A national cohort study on pediatric Behçet's disease: cross-sectional data from an Italian registry. Pediatr Rheumatol Online J. 2017 Dec 21;15(1):84. doi: 10.1186/s12969-017-0213-x. Erratum in: Pediatr Rheumatol Online J. 2018 Apr 23;16(1):29. PMID: 29268757; PMCID: PMC5740899. 7) Maggio MC, Corsello G, Prinzi E, Cimaz R. Kawasaki disease in Sicily: clinical description and markers of disease severity. Ital J Pediatr. 2016 Nov 2;42(1):92. doi: 10.1186/s13052-016-0306-z. PMID: 27806720; PMCID: PMC5094032. 8) Maggio MC, Cimaz R, Failla MC, Dones P, Corsello G. Typical Kawasaki disease with atypical pneumonia: a paediatric case report. Scand J Rheumatol. 2021 May;50(3):248-249. doi: 10.1080/03009742.2020.1784458. Epub 2020 Aug 17. PMID: 32804000. 9) Maggio MC, Cimaz R, Alaimo A, Comparato C, Di Lisi D, Corsello G. Kawasaki disease triggered by parvovirus infection: an atypical case report of two siblings. J Med Case Rep. 2019 Apr 24;13(1):104. doi: 10.1186/s13256-019-2028-5. PMID: 31014402; PMCID: PMC6480815. 10) Islam M, Chou M, Braithwaite T, Siddiqui A. Bilateral anterior non-necrotising scleritis, anterior uveitis, and unilateral facial nerve palsy in paediatric inflammatory multisystem syndrome temporally associated with COVID-19. Lancet Rheumatol. 2021 Nov;3(11):e818. doi: 10.1016/S2665-9913(21)00272-1. Epub 2021 Sep 13. PMID: 34541553; PMCID: PMC8437677.

Authors:

Maria Cristina Maggio Professor 1; Filippo Collura M.D. 1; Maria Michela D'Alessandro M.D. 2; Barbara Gramaglia, M.D. 1; Giovanni Corsello Full Professor 1.

1) University Department PROMISE "G. D'Alessandro, University of Palermo - Children Hospital "G. Di Cristina", A.R.N.A.S. Palermo, Italy

2) U.O.C. of Paediatric Nephrology, Children Hospital "G. Di Cristina", A.R.N.A.S. Palermo, Italy 